\title{
Amphiphilic core crosslinked star polymers for the delivery of hydrophilic drugs from hydrophobic matrices
}

Katarzyna Somszor, ${ }^{a}$ Stephanie Allison-Logan, ${ }^{\mathrm{a}, \mathrm{b}}$ Fatemeh Karimi, ${ }^{\mathrm{b}, \mathrm{c}}$ Thomas McKenzie, ${ }^{\mathrm{b}}$ Qiang $\mathrm{Fu},{ }^{\mathrm{b}}$ Andrea O'Connor, ${ }^{\mathrm{a}}$ Greg Qiao*b and Daniel Heath*a

${ }^{a}$ Department of Biomedical Engineering, University of Melbourne, Parkville 3010 VIC, Australia

${ }^{\mathrm{b}}$ Department of Chemical Engineering, University of Melbourne, Parkville 3010 VIC Australia

${ }^{\mathrm{c}}$ Graduate School of Biomedical Engineering, University of New South Wales, Sydney, NSW 2052, Australia

\section{Supporting Information}

\begin{abstract}
Materials
$\varepsilon$-Caprolactone (97\%), methane sulfonic acid (MSA, 98\%), copper (II) bromide $\left(\mathrm{CuBr}_{2}\right)$, poly(ethylene glycol) diacrylate $(\mathrm{Mn}=250 \mathrm{Da}$, PEGDA), poly(ethylene glycol) methyl ether acrylate $(\mathrm{Mn}=480 \mathrm{Da}, \mathrm{PEGA}), 2$-hydroxyethyl acetate, heparin sodium salt from porcine mucosa (Grade I-A, $\geq 180$ USP units/mg), Azure A chloride, poly( $\varepsilon$-caprolactone) pellets (70-90 kDa), and $N, N$-dimethylformamide (DMF) were purchased from Sigma Aldrich. Dimethyl sulfoxide (99\%, DMSO) was purchased from Chem-Supply. Graphene oxide $\left(5 \mathrm{mg} \mathrm{mL}^{-1}\right.$ ethanol) was supplied by ACS Materials LLC. Dry toluene, dichloromethane and $\varepsilon$-caprolactone were obtained by distillation from calcium hydride before use. Poly(ethylene glycol) diacrylate, $\mathrm{Mn}=250 \mathrm{Da}$, and poly (ethylene glycol)methyl ether acrylate $(\mathrm{Mn}=480 \mathrm{Da})$ were deinhibited by passing through basic alumina columns before use. A Beaufly Nail Lamp was used as the UV source $\left(36 \mathrm{~W}, \lambda_{\max }=365 \mathrm{~nm}\right)$.

2-Hydroxyethyl $\alpha$-bromoisobutyrate (HEBriB) was synthesized via the esterification of 1,2ethanediol with $\alpha$-bromoisobutyryl bromide (98\%, Aldrich), purified by flash column chromatography, and characterized by ${ }^{1} \mathrm{H}$ NMR and ${ }^{13} \mathrm{C}$ NMR. Tris[2-(diethylamino)ethyl]amine (Me ${ }_{6}$ TREN) was synthesized according to the procedure reported in the literature. ${ }^{1}$
\end{abstract}

\section{Characterization}

\section{Gel Permeation Chromatography}

Molecular weight characterizations were carried out via gel permeation chromatography (GPC) using DMF as the mobile phase unless otherwise stated. The GPC analysis was conducted on a Shimadzu liquid chromatography system equipped with a Shimadzu RID-10 refractometer $(\lambda=633 \mathrm{~nm})$, and Shimadzu SPD-20A UV-vis detector using three identical Jordi columns $(5 \mu \mathrm{m}$ bead size, Jordi Gel Fluorinated DVB Mixed Bed) in series operating at $50{ }^{\circ} \mathrm{C}$. DMF (>99\%, Aldrich) with $0.05 \mathrm{M} \mathrm{LiBr}$ was employed as the mobile phase at a flow rate of $1 \mathrm{~mL} \mathrm{~min}{ }^{-1}$ following previously described protocols. Calibration curves generated from poly (methyl methacrylate) (PMMA) standards were used to determine molecular weights of the synthesized polymers. 
When aqueous-phase GPC was employed, a separate Shimadzu liquid chromatography system was utilized, equipped with a Shimadzu RID-10 refractometer $(\lambda=633 \mathrm{~nm})$, using three Waters Ultrahydrogel columns in series ((i) $250 \AA$ porosity, $6 \mu \mathrm{m}$ diameter bead size; (ii) and (iii) linear, $10 \mu \mathrm{m}$ diameter bead size), operating at room temperature. The eluent was milli-Q water containing $0.1 \% \mathrm{v} / \mathrm{v}$ trifluoroacetic acid (TFA) at a flow rate of $1 \mathrm{~mL} \mathrm{~min}{ }^{-1}$. The molecular weight characteristics of the analyte were determined by a comparison to molecular weight distribution poly(ethylene glycol) standards. All samples were filtered through $0.45 \mu \mathrm{m}$ nylon filters prior to injection.

Proton Nuclear Magnetic Resonance ( ${ }^{1} \mathrm{H}$ NMR) spectroscopy was conducted on a Varian Unity $400 \mathrm{MHz}$ spectrometer operating at $400 \mathrm{MHz}$, using the solvent chloroform $\left(\mathrm{CHCl}_{3}\right)$ (Cambridge Isotope Laboratories) as reference and sample concentrations of approximately $10 \mathrm{mg} \mathrm{mL}^{-1}$.

Dynamic Light Scattering (DLS) was performed using a Malvern Zetasizer. Spectra were obtained at concentrations of $1 \mathrm{mg} \mathrm{mL}^{-1}$ in water at $25^{\circ} \mathrm{C}$.

\section{Additional Information}

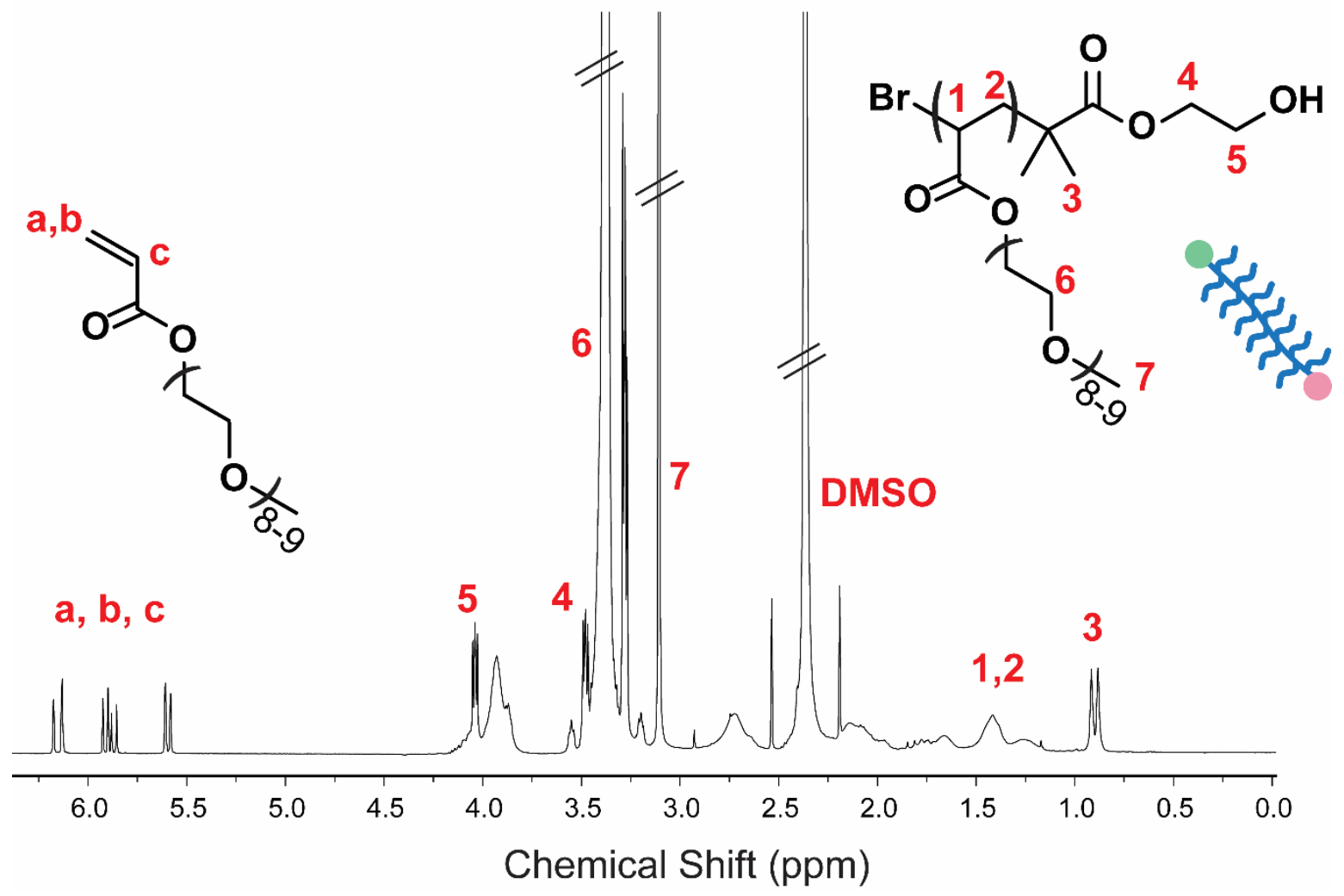

Figure S1. Representative ${ }^{1}$ HNMR spectrum of PPEGA at low conversion, with proton peaks corresponding to both polymer and monomer assigned. 

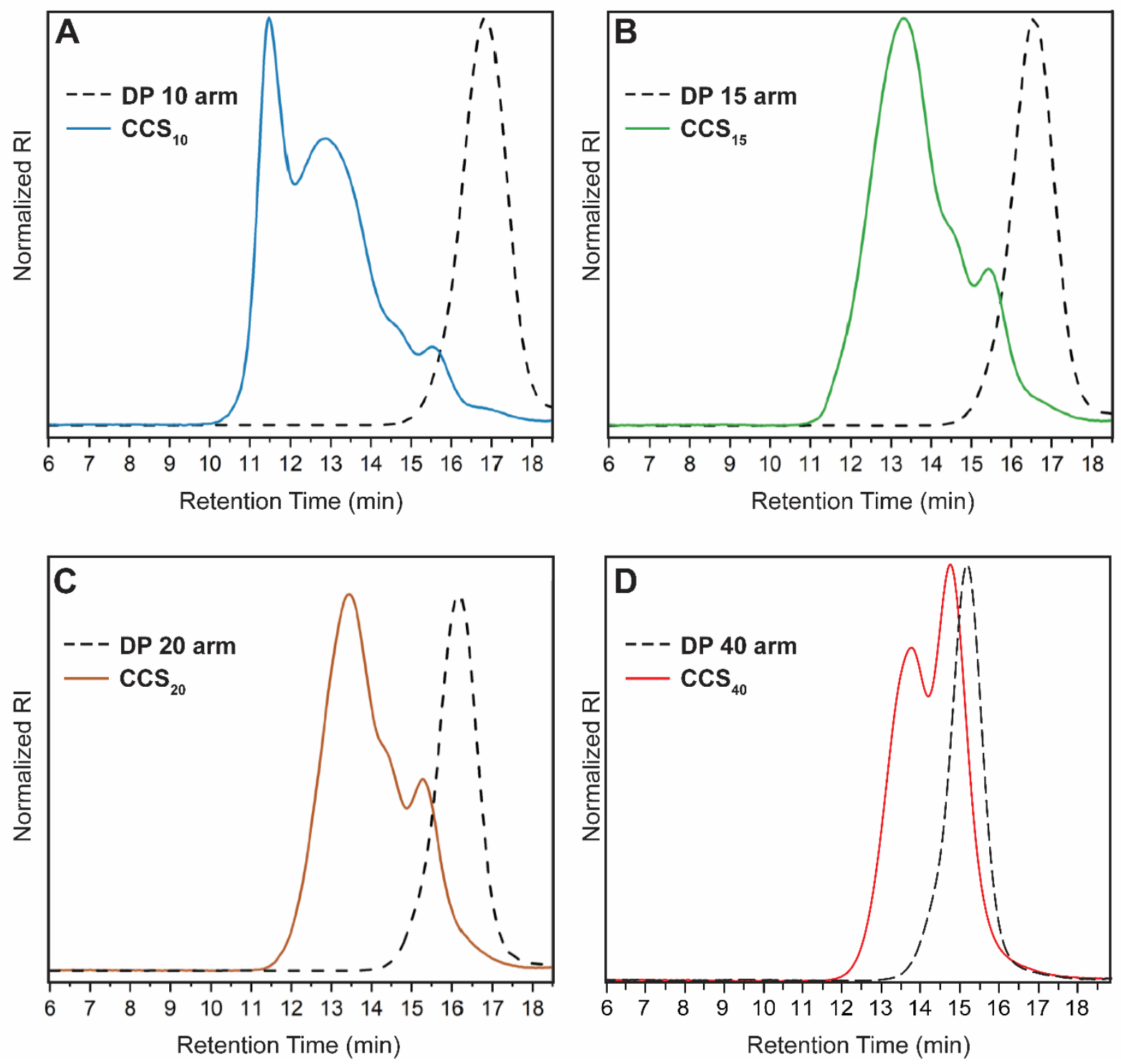

Figure S2. Gel permeation chromatograms of unpurified CCS formed from PPEGA brush arms with DP A) 10, B) 15 , C) 20 , and D) 40. PEGDA was used as a crosslinking agent, with an arm:crosslinker ratio of $1: 15$. 


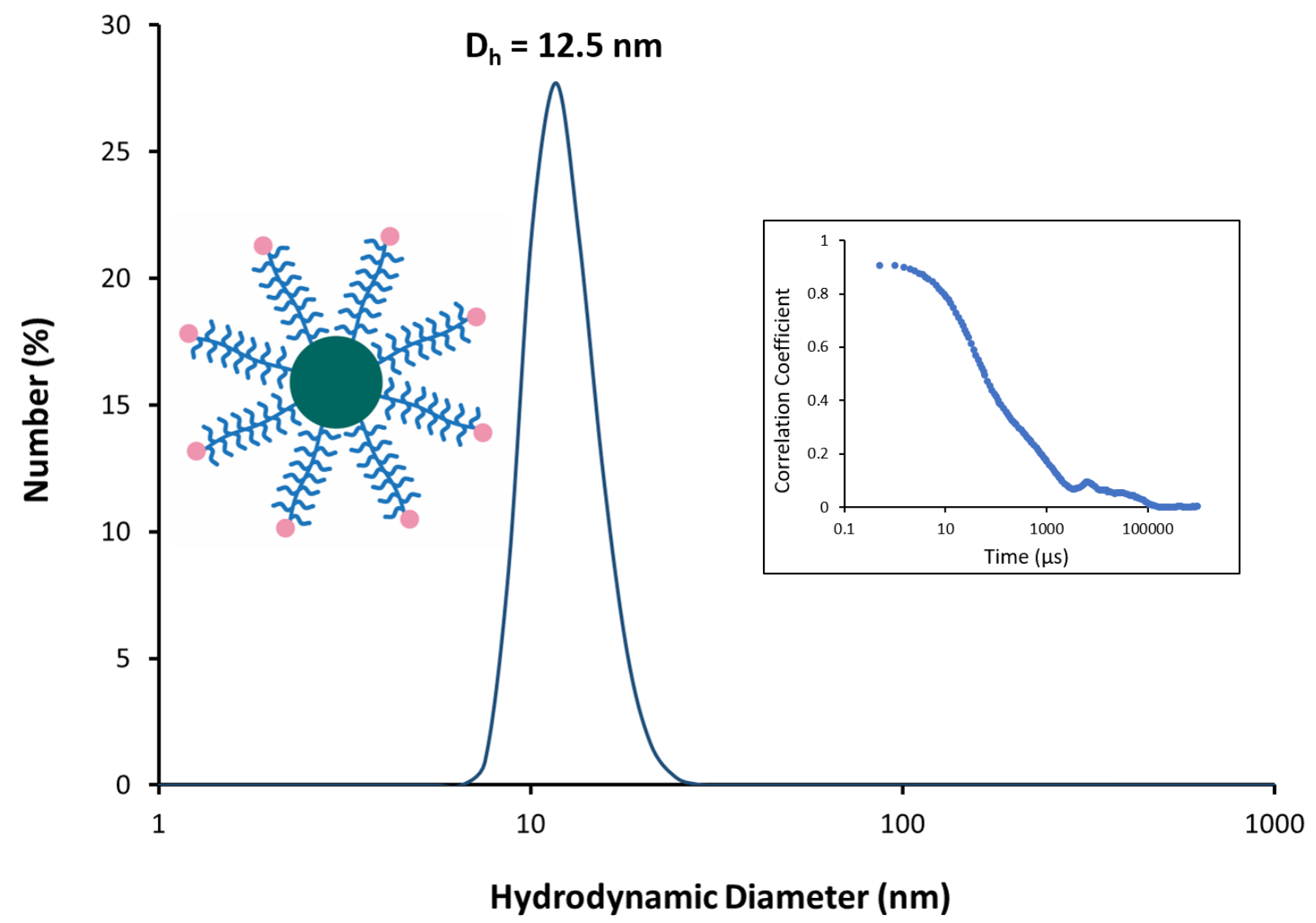

Figure S3. Dynamic light scattering analysis of PPEGA 20 in water demonstrated a hydrodynamic diameter of $12.5 \mathrm{~nm}$. 


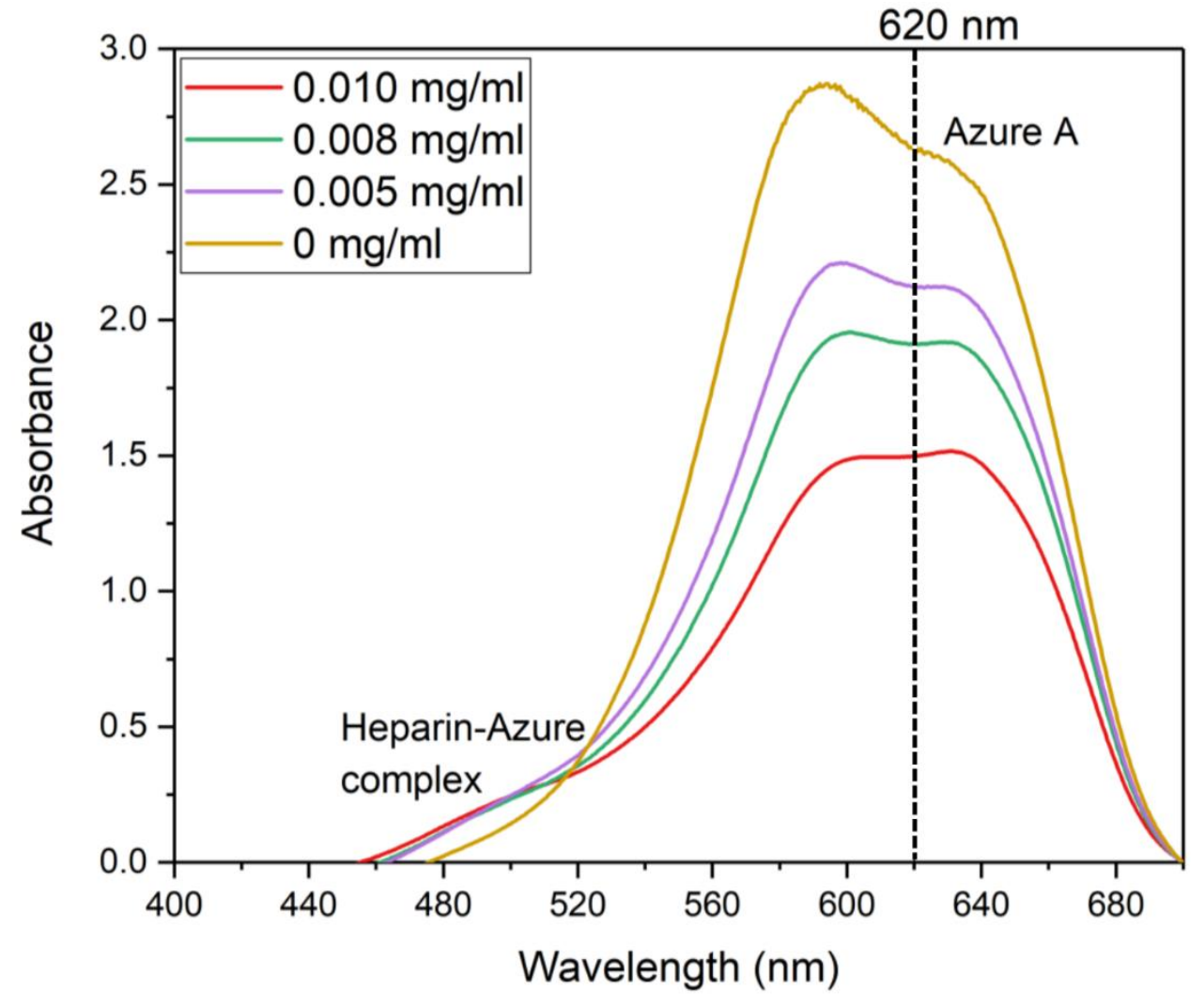

Figure S4. UV-Vis absorbance spectra of Azure A with increasing concentrations of heparin. The absorbance of Azure A alone is shown in the $0 \mathrm{mg} / \mathrm{mL}$ heparin solution. The absorption band at $620 \mathrm{~nm}$ decreases with increasing heparin concentration as a heparin-Azure A complex forms, resulting in a loss of Azure-A colour. Known concentrations of heparin were added to Azure A and the absorbance at $620 \mathrm{~nm}$ was measured to generate a standard curve for heparin concentration. 
A

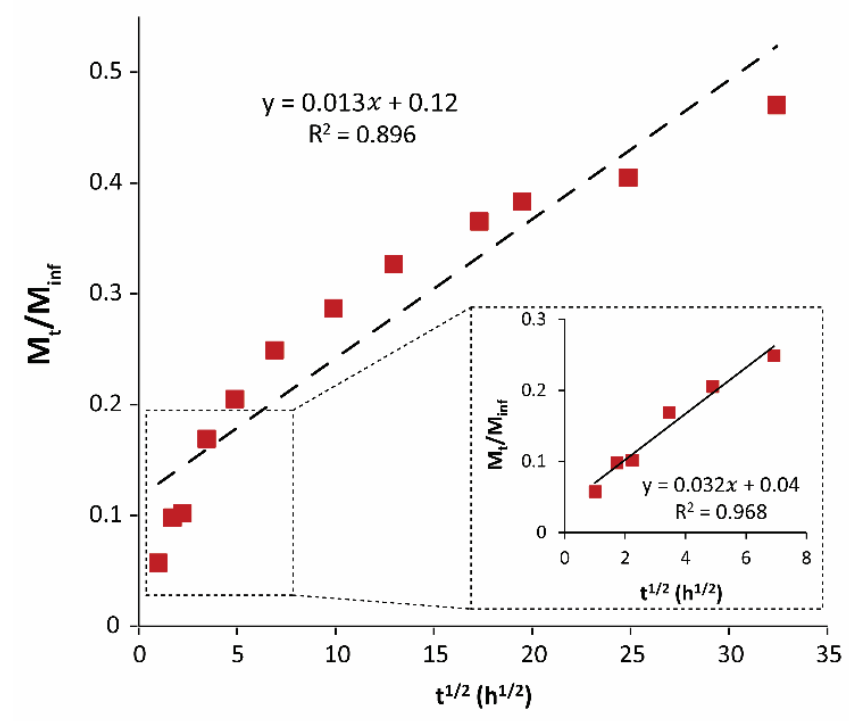

B

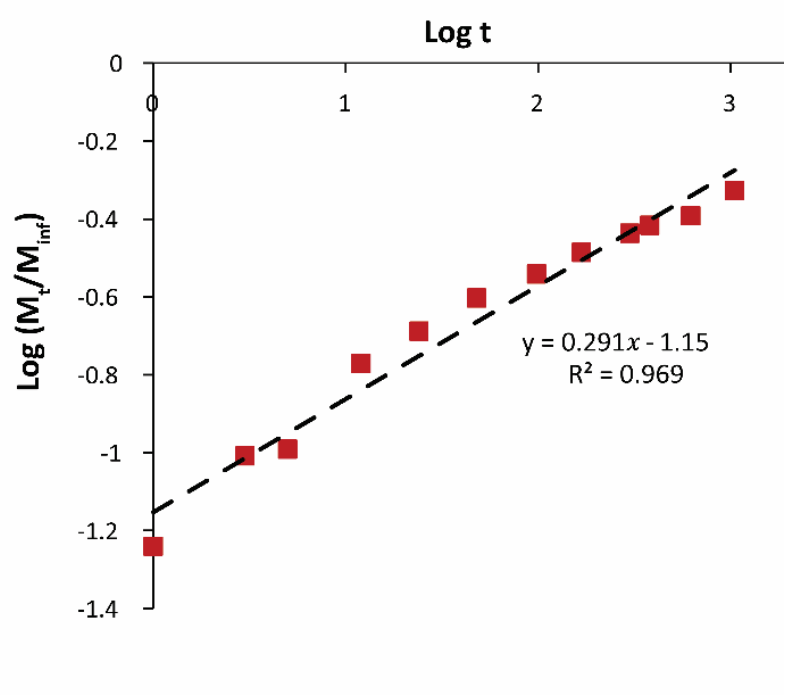

Figure S5. Modelling of heparin released from the PCL-rGO matrix containing $10 \mathrm{wt} \%$ hep-CCS-PCL. A) The Higuchi model is derived from Fick's second law of diffusion. B) The Korsmeyer-Peppas or power model. For both models, $M_{t}$ represents the cumulative amount of heparin released at time $t$ of the total amount of heparin loaded, $\mathrm{M}_{\mathrm{inf}}$. Data points from in vitro release are shown as red squares while the black dashed line demonstrates the line of best fit.

Higuchi Model Equation:

$$
\left(M_{t} / M_{\text {inf }}\right)=k t^{1 / 2}
$$

Korsmeyer-Peppas Equation²:

$$
\left(M_{t} / M_{\text {inf }}\right)=k t^{n}
$$

The equation was converted to its logarithmic form to give a linear plot, from which the exponent, $n$, was found ${ }^{3}$ :

$$
\log \left(M_{t} / M_{\text {inf }}\right)=\log k+n \log t
$$




\section{References}

(1) Ciampolini, M.; Nardi, N. Five-coordinated high-spin complexes of bivalent cobalt, nickel, and copper with tris (2-dimethylaminoethyl) amine. Inorg. Chem. 1966, 5 (1), 41.

(2) Korsmeyer, R. W.; Gurny, R.; Doelker, E.; Buri, P.; Peppas, N. A. Mechanisms of solute release from porous hydrophilic polymers. Int. J. Pharm. 1983, 15 (1), 25.

(3) Sinclair, G. W.; Peppas, N. A. Analysis of non-Fickian transport in polymers using simplified exponential expressions. J. Membr. Sci. 1984, 17 (3), 329. 